\title{
Tkachenko modes as sources of quasiperiodic pulsar spin variations
}

\author{
Jorge Noronhat \\ Frankfurt Institute for Advanced Studies, J. W. Goethe-Universität, D-60054 Frankfurt am Main, Germany \\ Armen Sedrakian丹 \\ Institute for Theoretical Physics, J. W. Goethe-Universität, D-60054 Frankfurt am Main, Germany
}

\begin{abstract}
We study the long wavelength shear modes (Tkachenko waves) of triangular lattices of singly quantized vortices in neutron star interiors taking into account the mutual friction between the superfluid and the normal fluid as well as the shear viscosity of the normal fluid. The set of Tkachenko modes that propagate in the plane orthogonal to the spin vector are weakly damped if the coupling between the superfluid and the normal fluid is small. In strong coupling, their oscillation frequencies are lower and are undamped for small and moderate shear viscosities. The periods of these modes are consistent with the observed $\sim 100-1000$ day variations of spin for PSR 1828-11.
\end{abstract}

\section{INTRODUCTION}

During the last decade mounting evidence has emerged for the existence of long-period oscillations in a handful of pulsars [1, 2, 3, 4, 5]. An outstanding example of this kind of phenomenon is observed in PSR 1828-11 [3]. Its timing residuals are modulated with periods of 256 and 511 days, while a 1009-day periodicity is inferred with lower confidence. These timing residuals coincide with periodic modulations of the pulse shape, which is a strong indication for the precessional motion(s) of the pulsar with the periods quoted above [3]. In fact, various models of precessing neutron stars $[6,7,8,8,10]$ fit the timing data fairly well. However, it is known that free precession is incompatible with the existence of a superfluid in the pulsar's interior if the superfluid is strongly coupled to the normal fluid [11, 12, 13, 14]. The mutual friction for the superfluids present in the crust and core of neutron stars, which are derived using microscopic calculations, cover a broad range of values. A reliable calculation necessarily involves the superconducting and superfluid properties of the fluid(s) and the non-superconducting material, which resists to vortex motion. While precession remains a viable model for the quasiperiodic oscillations observed in pulsar timing data, here we follow a different route [15] by exploring the propagation of Tkachenko modes in pulsars as the source of long term variations.

Charge neutral superfluids in neutron star interiors rotate by forming an array of singly quantized vortices. In their lowest energy state the vortices form a twodimensional triangular lattice. The lattice supports collective elastic modes, Tkachenko waves, in which the vortices are displaced parallel to each other 16, 17, 18, 19]. Their undamped propagation would lead to variations of the angular momentum of the superfluid due to the lo-

\footnotetext{
*Electronic address: noronha@fias.uni-frankfurt.de

${ }^{\dagger}$ Electronic address: sedrakian@th.physik.uni-frankfurt.de
}

cal variations of the density of the vortex lines as well as periodic variations in the rotation and spin-down speeds of the star.

Ruderman [20] pointed out that the frequency of the Tkachenko modes is of the order of several hundred days and these modes could be responsible for the quasiperiodic timing residuals observed in the Crab pulsar. Little attention has been paid to the role played by the Tkachenko modes in neutron stars since Ruderman's 1970 paper. Here we reinvestigate the propagation of Tkachenko modes in neutron star superfluids and calculate how these modes are damped by mutual friction and the shear viscosity of normal matter. These factors are clearly important for the continuous propagation of these modes, which would certainly lead to observable effects. Provided that the observed variations are indeed caused by the shear modes of the lattice we can ask the question: what do the quasi-sinusoidal variations tell us about the microscopic physics governing superfluids in neutron stars?

This question can be answered by describing the physics present at micro- and mesoscopic scales (the upper limit on these scales is set by the size of the neutron vortex) in terms of the few parameters that enter the equations of superfluid hydrodynamics. These parameters include the kinetic coefficients and other local characteristics (e. g., density, baryon and lepton fractions, etc). The predictions obtained within this hydrodynamic description can be presented in a form that is independent of the details of physics at the micro- and mesoscopic scales.

In this paper we pursue this "model independent" approach, although some comments about the physics present at intermediate scales are in order. A key problem concerning the crust of neutron stars is the question of whether neutron vortices are pinned to the crustal nuclei both under static and dynamical conditions. The static problem always has pinning solutions for infinitesimally attractive interactions between a neutron vortex and a nucleus (for repulsive interactions the vortex is localized in between the lattice sites) [21, 22]. The dynamical problem of repinning under the action of exter- 
nal superflow [23] allows for repinning solutions only for strong pinning potentials and current estimates for the pinning energy do not favor repinning solutions. However, if the axisymmetry of the problem is lost, as in the case of precessing neutron stars, the vortices may not pin at all [24].

Another issue concerning neutron star crusts is that they may often undergo starquakes [25, 26], which drive the vortex lattice out of its equilibrium state. In fact, the form of the lattice may change from a triangular shape into some other structure reflecting the symmetries of the underlying nuclear lattice. Moreover, other possible scenarios include cases where the vortices may acquire multiple quanta of circulation or the lattice may contain impurities and vacancies. Thus, our working assumption that the ground state of the lattice consists of a simple triangular array of vortices may not be applicable to the crusts of frequently quaking neutron stars.

Regarding the cores of neutron stars, a major question is whether the proton fluid is a type-I [27, 28, 29] or type-II superconductor $30,31,32,33,34,35,36,37$, 38, 39, 40] (it may also become unpaired at high densities). The friction in the case of type-II superconductors is large [38], which implies that precession is impossible. If, however, the proton fluid is a type-I superconductor the friction is found to be small and compatible with precession [15].

Finally, we would like to point our that the superfluids may develop quantum turbulence when the velocity difference between the superfluid and the normal fluid exceeds a certain critical value. Turbulent states in superfluid He may be generated in many ways, e. g., by driving the normal fluid along the vortex lines through a temperature gradient. Neutron stars superfluids may also develop turbulent states [41, 42, 43], which could be caused either by unstable precession [44] or tectonic activity in the crusts [45].

This paper is organized as follows. In Sec. II we recapitulate the equations of superfluid hydrodynamics that include the combined effects of vortex tension, mutual friction, and shear viscosity. Sec. IIII is devoted to the derivation of the characteristic equation for the Tkachenko and inertial modes and their numerical study. Our conclusions are summarized in Sec. IV.

\section{SUPERFLUID HYDRODYNAMICS WITH VORTEX TENSION}

It is convenient at this point to discuss the three main length scales that appear in our study in more detail. The relevant length scales are the vortex core radius $\sim 10^{-12} \mathrm{~cm}$, the intervortex spacing $\sim 10^{-3} \mathrm{~cm}$, and the size of the superfluid phase $\sim 10^{5} \mathrm{~cm}$ (we refer to these three different scales as micro, meso, and macroscopic scales). A hydrodynamic description requires averaging over the mesoscopic scales. In order to describe the deformations of the vortex lattice, one needs an ad- ditional dynamical variable, the local deformation of the vortex lattice $\boldsymbol{\epsilon}(\mathbf{r})$, which can be incorporated into the Bekarevich-Khalatnikov superfluid hydrodynamics [46]. A hydrodynamic description of superfluids that includes lattice deformations has been studied by a number of authors [47, 48, 49, 50] and in this work we use the Baym-Chandler version of superfluid hydrodynamics [49] to study the Tkachenko modes and their damping due to mutual friction and shear viscosity.

The fluid motions are naturally separated into the center of mass motion (known as first sound) and second sound, which corresponds to temperature dependent relative oscillations between the superfluid and the normal component. These motions are conveniently described in terms of the total mass current $\mathbf{j}=\rho_{N} \mathbf{v}_{N}+\rho_{S} \mathbf{v}_{S}$ and the relative velocity $\mathbf{w}=\mathbf{v}_{N}-\mathbf{v}_{S}$, where $\rho_{S}\left(\rho_{N}\right)$ and $\mathbf{v}_{S}\left(\mathbf{v}_{N}\right)$ are the superfluid (normal fluid) density and velocity, respectively. In addition, one needs an equation for the time variations of the lattice deformation $\boldsymbol{\epsilon}(\mathbf{r})$. We note in passing that $\rho=\rho_{N}+\rho_{S}$ is the total mass density and the tiny effects arising due to the inertia of the vortex lines are neglected.

The linearized version of the fundamental superfluid hydrodynamic equations written for the net mass current, the relative velocity, and the superfluid velocity are

$$
\begin{aligned}
& \frac{\partial \mathbf{j}}{\partial t}+(2 \boldsymbol{\Omega} \times \mathbf{j})+\mathbf{C}+\boldsymbol{\sigma}+\boldsymbol{\nabla} P+\rho \boldsymbol{\nabla} \phi=0, \\
& \frac{\partial \mathbf{w}}{\partial t}+(2 \boldsymbol{\Omega} \times \mathbf{w})-\frac{\boldsymbol{\sigma}}{\rho_{S}}-\mathbf{f}=0, \\
& \frac{\partial \mathbf{v}_{S}}{\partial t}+\left(2 \boldsymbol{\Omega} \times \frac{\partial \boldsymbol{\epsilon}}{\partial t}\right)+\frac{\boldsymbol{\nabla} P}{\rho}+\boldsymbol{\nabla} \phi=0,
\end{aligned}
$$

where $\boldsymbol{\Omega}=(0,0, \Omega)$ is the spin vector ( $\Omega$ is the pulsar rotation frequency), $P=P_{0}-\rho(\boldsymbol{\Omega} \times \mathbf{r})^{2} / 2, P_{0}$ is the pressure in the fluid at rest, and $\boldsymbol{\sigma}$ is the vortex elastic force density defined as

$$
\boldsymbol{\sigma}=\mu_{S}\left[2 \boldsymbol{\nabla}_{\perp} \cdot\left(\boldsymbol{\nabla}_{\perp} \cdot \boldsymbol{\epsilon}\right)-\nabla_{\perp}^{2} \boldsymbol{\epsilon}\right]-2 \Omega \lambda \frac{\partial^{2} \boldsymbol{\epsilon}}{\partial z^{2}},
$$

where $\nabla_{\perp}$ is the gradient in the $x-y$ plane and $\mu_{S}=$ $\rho_{S} \hbar \Omega / 8 m_{N}$ is the shear modulus of the triangular vortex lattice calculated by Tkachenko [16, 17, 18, 19]. Moreover, $m_{N}$ is the bare neutron mass and the vortex tension is given by

$$
\lambda=\frac{\hbar \rho_{S}}{8 m_{N}} \ln \left(\frac{b}{a}\right),
$$

where $a$ is the coherence length and $b=\left(\pi \hbar / \sqrt{3} m_{N} \Omega\right)$ is the vortex radius of the triangular lattice. The Newtonian gravitational potential $\phi$ satisfies the equation

$$
\nabla^{2} \phi=\nabla^{2}\left(\phi_{S}+\phi_{N}\right)=4 \pi G\left(\rho_{S}+\rho_{N}\right),
$$

where $G$ is the Newton's constant and $\phi_{S}$ and $\phi_{N}$ are the gravitational potentials of the superfluid and the normal 
fluid, respectively. The force density $\mathbf{C}$ is defined as $C_{i}=$ $\nabla_{k} \tau_{i k}$, where $\tau_{i k}$ is the viscous stress tensor

$$
\tau_{i k}=-\eta\left(\nabla_{i} v_{N k}+\nabla_{k} v_{N i}-\frac{2}{3} \delta_{i k} \boldsymbol{\nabla} \cdot \mathbf{v}_{N}\right)
$$

whereas $\eta$ is the shear viscosity. Note that we do not take into account the effects from bulk viscosity and thermal conductivity. Finally, the mutual friction force is

$$
\begin{aligned}
\mathbf{f}=\beta \rho_{S}[\mathbf{n} \times[\boldsymbol{\omega} & \left.\left.\times\left(\frac{\partial \boldsymbol{\epsilon}}{\partial t}-\mathbf{v}_{N}\right)\right]\right] \\
& +\beta^{\prime} \rho_{S}\left[\boldsymbol{\omega} \times\left(\frac{\partial \boldsymbol{\epsilon}}{\partial t}-\mathbf{v}_{N}\right)\right],
\end{aligned}
$$

where $\boldsymbol{\omega}=\boldsymbol{\nabla} \times \mathbf{v}_{S}$ is the quantum circulation vector, $\mathbf{n} \equiv \boldsymbol{\omega} / \omega$, and $\beta$ and $\beta^{\prime}$ are the phenomenological mutual friction coefficients.

The dissipative terms in the hydrodynamic equations defined above (such as the mutual friction forces) are the most general expressions that can be used in the description of a rectilinear vortex lattice in equilibrium that are still compatible with the conservation laws and the assumption that the dissipative function is a positive quadratic form of the perturbations (higher order terms are neglected). Furthermore, we would like to remark that Baym-Chandler hydrodynamics only describes the linear order corrections in the lattice displacements, which are considered to be small. The neutron vortex lattice in neutron stars may, however, become unstable towards forming a tangle with turbulent superfluid flow, which would require a thorough revision of the hydrodynamic equations. In particular, the form of the mutual friction force (8) should be changed to the one suggested by Groter and Mellink [51] where $\mathbf{f} \propto \mathbf{w}$. These aspects of superfluid dynamics are beyond the scope of this work (see Refs. [41, 42, 43]).

\section{OSCILLATION MODES}

We consider plane wave perturbations with respect to the equilibrium, which corresponds to uniform rotation. We use a Cartesian system of coordinates where the $z$ axis is directed along the spin vector $\boldsymbol{\omega}$. The vectors $\mathbf{j}$ and $\mathbf{w}$ can be decomposed into transverse and longitudinal parts, i.e., $\mathbf{j}=\mathbf{j}_{t}+\mathbf{j}_{l}$ and $\mathbf{w}=\mathbf{w}_{t}+\mathbf{w}_{l}$. The transverse parts we are interested in satisfy the condition

$$
\nabla \cdot \mathbf{j}_{t}=\nabla \cdot \mathbf{w}_{t}=0
$$

The perturbation equation for the transverse components of the vectors $\mathbf{j}$ and $\mathbf{w}$ derived from Eqs. (1)-(3) are (hereafter the subscript $t$ is suppressed)

$$
\begin{aligned}
\frac{\partial j_{i}}{\partial t}+\left(2 \epsilon_{l m n} \Omega_{m} j_{n}+\sigma_{l}+k_{m} \tau_{l m}\right) P_{i l} & =0 \\
\frac{\partial w_{i}}{\partial t}+\left(2 \epsilon_{l m n} \Omega_{m} w_{n}-\frac{\sigma_{l}}{\rho_{S}}-f_{l}\right) P_{i l} & =0 \\
\frac{\partial v_{i}}{\partial t}+2 \epsilon_{l m n} \Omega_{m} \frac{\partial \epsilon_{n}}{\partial t} P_{i l} & =0 .
\end{aligned}
$$

In the equations above we used the projector $P_{i l}=$ $\delta_{i l}-k_{i} k_{l} / k^{2}$ where $\mathbf{k}$ is the wave vector. Our coordinate system is such that the wave vector lies on the $z-x$ plane, i.e., $\mathbf{k}=(k \sin \theta, 0, k \cos \theta)$ where $\theta$ is the angle formed by the vectors $\boldsymbol{\Omega}$ and $\mathbf{k}$.

Writing the time perturbations as $j_{i}(t) \sim j_{i} e^{2 \Omega p t}$ (a similar definition is used for the other vectors) we obtain, after some algebra, the characteristic equation $\operatorname{det}\left\|K_{i j}\right\|=0$ where

$$
K_{i j}=\left(\begin{array}{cccc}
p-\tilde{\eta} \alpha d & \left(\gamma_{S} h-1\right) & -\tilde{\eta} \gamma_{S} \alpha d & -\gamma_{S} \gamma_{N} h \\
d+\gamma_{S} g & p-\tilde{\eta} & \gamma_{S} \gamma_{N} g & -\tilde{\eta} \gamma_{S} \\
-\hat{\beta} g & -\hat{\beta}^{*} h & p+\hat{\beta}\left(d+\gamma_{N} g\right) & -\hat{\beta}^{*}\left(1-\gamma_{N} h\right) \\
-\hat{\beta}^{*} g & \hat{\beta} h & \hat{\beta}^{*}\left(d+\gamma_{N} g\right) & p+\hat{\beta}\left(1-\gamma_{N} h\right)
\end{array}\right)
$$

We used the following shorthand notations in the definition of the matrix $K_{i j}: \gamma_{N / S}=\rho_{N / S} / \rho, d^{1 / 2}=\cos \theta$, $\hat{\beta}^{*}=1-\hat{\beta}^{\prime}, \tilde{\eta}=\eta k^{2} /(2 \Omega \rho), \alpha=(4-d) / 3, \hat{\beta}=\gamma_{N}^{-1} \beta$, and $\hat{\beta}^{\prime}=\gamma_{N}^{-1} \beta^{\prime}$. Moreover, we defined

$$
\begin{aligned}
& g=\frac{k^{2}}{4 \Omega^{2} \rho_{S}}\left[\mu_{S}-d\left(\mu_{S}-2 \Omega \lambda\right)\right], \\
& h=\frac{k^{2}}{4 \Omega^{2} \rho_{S}}\left[\mu_{S}-d\left(\mu_{S}+2 \Omega \lambda\right)\right] .
\end{aligned}
$$

Note that the coefficients $g$ and $h$ are independent of the density because $\mu_{S} \sim \rho_{S}$ and $\lambda \sim \rho_{S}$. The density appears only through the normalization of the shear viscosity $\tilde{\eta} \sim \rho^{-1}$. The eigenmodes of the matrix in Eq. (13) provide the oscillatory modes in the general case where the shear viscosity of normal matter and the mutual friction are included. In the non-dissipative limit $\left(\beta=\beta^{\prime}=\eta=0\right)$ the modes separate into two independent sets that describe the inertial and Tkachenko modes, respectively. The (real) eigenfrequencies of these modes in units of $2 \Omega$ are

$$
p_{I}= \pm i d^{1 / 2}, \quad p_{T}= \pm i[(d+g)(1-h)]^{1 / 2},
$$




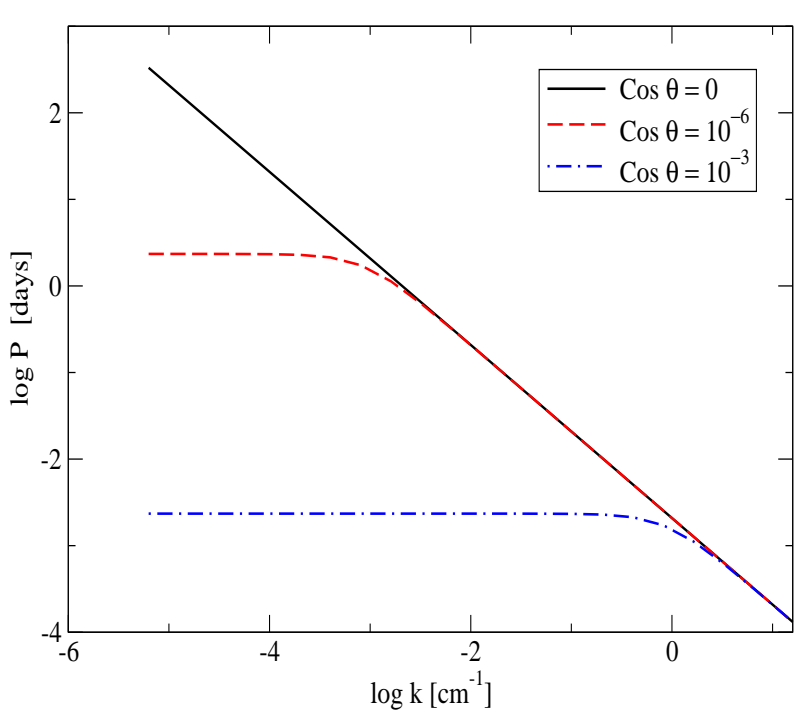

FIG. 1: (Color online) Dependence of the period $P=2 \pi /\left|p_{T}\right|$ of the Tkachenko modes on the wave vector for $d=0$ (solid black line), $d=10^{-12}$ (dashed red line), and $d=10^{-6}$ (dashed-dotted blue line). For large wavelengths the periods are on the order of 100 days. In particular, for $\Omega_{\star}=15.51$ Hz we obtain that $P\left(k_{\min }, d=0\right)=331$ days if the core size is $R_{c}=10 \mathrm{~km}$ and 256 days when $R_{c}=7.7 \mathrm{~km}$.

where the indices $I$ and $T$ refer to inertial and Tkachenko modes, respectively.

If the Tkachenko modes are generated within superfluid shells with the width of $R_{c} \sim 10 \mathrm{~km}$ their corresponding wave vectors are of the order of $k_{\min }=2 \pi / R_{c} \sim$ $6.28 \times 10^{-6} \mathrm{~cm}$, which sets the lower limit on the wave vector. Since the hydrodynamic description breaks down at length scales $\sim 10 b\left(b \sim 10^{-3} \mathrm{~cm}\right.$ is the intervortex distance) the wave vector is bounded from above by the value $k_{\max }=73.3 \mathrm{~cm}^{-1}$. We are interested in the small wave vector limit $k \sim k_{\min }$ that describes vortex density waves across the entire superfluid shell.

The parameters $g$ and $h$ are of the order of $s=$ $\left(\hbar^{2} k_{\min }^{2} / 2 m_{n}\right)(8 \hbar \Omega)^{-1}$. For instance, when $k=k_{\min }$ and $\Omega_{\star}=15.51 \mathrm{~Hz}$ (the rotation frequency of PSR 1828-11) we obtain that $s=10^{-14}$. Therefore, for $s \ll 1$ the eigenmodes corresponding to Tkachenko waves in the dissipationless limit are given by $p_{T}= \pm i[(d+g)]^{1 / 2}$. In the limit $d \ll g$ where the wave vectors are highly collinear to the spin vector we obtain that $p_{T}= \pm i \sqrt{g}$ and, in the opposite limit $d \gg g$, the Tkachenko modes become identical to the inertial modes, i.e., $p_{T}=p_{I}$.

Fig. 1 displays the period of the Tkachenko modes without dissipation as a function of their wave vector. Only the long wavelength perturbations have periods on the order of 100 days, which are then relevant for observations. In this limit the periods rapidly decrease for perturbations with finite $d$. The period $P\left(k_{\min }, d=0\right)=331$ days for $\Omega_{\star}=15.51 \mathrm{~Hz}$ suggests that the shortest of the periods observed in PSR 1828-11, which corresponds to
256 days, should be identified with the fundamental oscillation mode. Oscillations with larger periods should then be identified with the higher-order harmonics of this mode. A period of 256 days can be obtained by adopting $R_{c}=7.7 \mathrm{~km}$, which translates into $k=8.16 \times 10^{-6}$ $\mathrm{cm}^{-1}$. This value is close to the upper limit for the size of any superfluid region inside a medium-heavy neutron star. It is important to remark that if the width of the superfluid region where Tkachenko waves can be found is at least one order of magnitude (or more) smaller than the values of $R_{c}$ quoted above the modes will be too fast to account for the long-period oscillations observed in PSR 1828-11 (as long as the renormalization effects due to mutual friction discussed below are neglected).

We now consider the effects of the shear viscosity and the mutual friction on the propagation of Tkachenko modes. It is convenient to use the drag-to-lift ratios $\zeta$ and $\zeta^{\prime}$ instead of $\beta$ and $\beta^{\prime}$ to describe mutual friction. These ratios are related by the following equations

$$
\beta=\frac{\zeta}{\left[\left(1-\zeta^{\prime}\right)^{2}+\zeta^{2}\right]}, \quad \beta^{\prime}=1-\frac{\beta\left(1-\zeta^{\prime}\right)}{\zeta} .
$$

Microscopic calculations indicate that $\zeta^{\prime} \simeq 0$. The limit $\zeta \rightarrow 0$ corresponds to weak coupling between the vortices and the normal fluid, while $\zeta \rightarrow \infty$ implies strong coupling.

Figure 2 shows the dependence of the modes derived from Eq. (13) on the drag-to-lift ratio $\zeta$ for several values of the shear viscosity and $d=0$. The value of $\tilde{\eta}$ is determined assuming a constant density of $3 \times 10^{14} \mathrm{~g} \mathrm{~cm}^{-3}$. In the limit where $\zeta$ and $\eta$ vanish we recover the results for the non-dissipative case discussed above. For $\eta=0$ the real part of the Tkachenko mode, which is doubly degenerate, vanishes only in a narrow window of values of $\zeta$. For larger values of $\zeta$, which corresponds to the strongly coupled region, the real part reaches an asymptotic value that is about $25 \%$ smaller than its value in the undamped limit. Note that in our plots only the regions where the modes change significantly are shown.

Assuming that the normal fluid is inviscid, the results in Fig. 2 imply that there are oscillations with even longer periods in the strongly coupled limit. The Tkachenko modes are significantly damped by mutual friction in the region where the real part vanishes. There the number of imaginary roots of the characteristic equation increases by one. Moreover, one of the imaginary roots is given by $\operatorname{Im} \omega=i \beta \Omega$, which continues beyond the figure's $y$ scale. This reflects the damping of the differential rotation between the superfluid and the normal fluid caused by mutual friction. This damping has no effect on the Tkachenko modes in strong coupling. For moderate values of viscosity $\left(\eta=5 \times 10^{17}\right.$ dyn s cm$\left.{ }^{-2}\right)$ the real part of the Tkachenko mode is reduced in the strongly coupled region. In this case, its imaginary part is smaller than the real part and, therefore, the oscillations are weakly damped. For large values of the shear viscosity $\left(\eta \sim 10^{19}\right.$ dyn $\mathrm{s} \mathrm{cm}^{-2}$ ) the real part of the Tkachenko mode vanishes in the strongly coupled limit. Finally, note that 


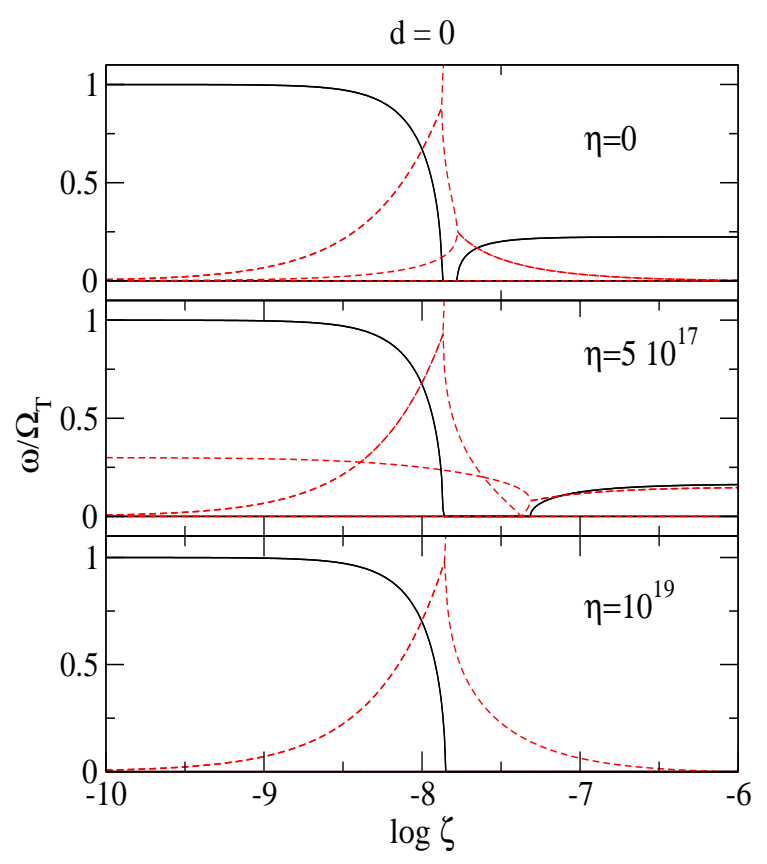

FIG. 2: (Color online) Dependence of the real (solid black line) and imaginary (dashed red line) parts of the Tkachenko modes $(\omega=i p)$ on the drag-to-lift ratio $\zeta$ for $\eta=0$ (upper panel), $\eta=7.5 \times 10^{17}$ (middle panel), and $\eta=1 \times 10^{19}$ (lower panel) in dyn $\mathrm{s} \mathrm{cm}^{-2}$ units. All modes are normalized by the non-dissipative value of the Tkachenko mode $\Omega_{T}=2 \pi / P$. The modes are computed taking $d=0$, which means that there are no inertial modes.

there are no inertial modes when $d=0$.

The modes when $d=10^{-14}$ are shown in Fig. 3. As discussed above, in the non-dissipative limit the Tkachenko and inertial modes coincide for sufficiently large $d$. For $\eta=0$ the modes can be distinguished in the strongly coupled limit because the Tkachenko mode vanishes for sufficiently large values of $\zeta$. When larger viscosities are considered $\left(\eta>10^{19}\right.$ dyn s cm$\left.{ }^{-2}\right)$ the difference between the real parts of the inertial and Tkachenko modes can be clearly resolved. If we increase $\eta$ even further we see that the real part of the inertial mode decreases and the imaginary part, which increases with $\eta$, becomes relevant. Finally, the real part of the inertial mode vanishes at $\eta \simeq 5 \times 10^{19}$ dyn $\mathrm{s} \mathrm{cm}^{-2}$.

The outer cores of neutron stars are mainly composed of light baryons, which pair in the isospin triplet states, and leptons. For densities of $2-3 \times 10^{14} \mathrm{~g} \mathrm{~cm}^{-3}$ and temperatures of $T \sim 10^{8} \mathrm{~K}$ the shear viscosity of the electron fluid was determined to be in the interval between $8-40 \times 10^{17}$ dyn s cm ${ }^{-2}$ [52]. This value of the temperature is a realistic upper bound on the temperature in the core of neutron stars except for very young objects such as the Vela and Crab pulsars. For colder stars the viscosity could be a few orders of magnitude

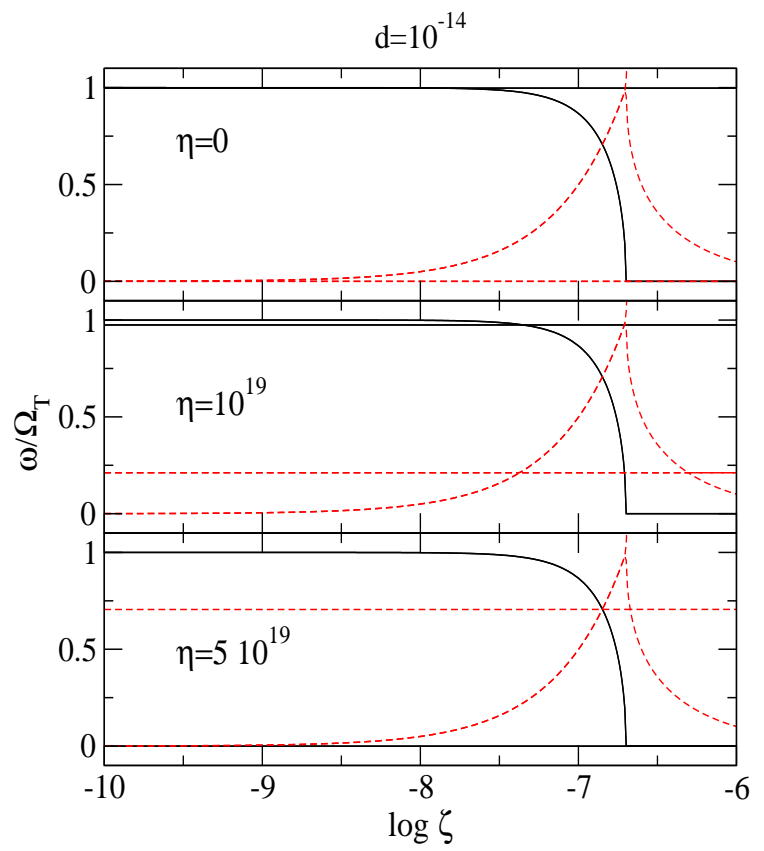

FIG. 3: (Color online) The same as in Fig. 2 in case of $d=$ $10^{-14}$ and $\eta=0$ (upper panel), $\eta=10^{19}$ (middle panel), and $\eta=5 \times 10^{19}$ (lower panel) in dyn s cm $\mathrm{cm}^{-2}$ units. Note that both the Tkachenko and inertial modes are displayed here.

larger because $\eta \sim T^{-2}$.

\section{CONCLUSIONS}

Our results indicate that Tkachenko modes are broadly consistent with the weakly coupled theories between the superfluid and the normal fluid, independent of the value the shear viscosity. The subclass with $d=\cos ^{2} \theta=0$ has periods that are consistent with the lowest observed periodicity in PSR 1828-11 of 256 days.

The existence of Tkachenko modes in the strongly coupled region depends on the shear viscosity of normal matter. For low viscosities the Tkachenko modes are (in strong coupling) renormalized to values that are a few times smaller than their non-dissipative limits. This implies that in strong coupling the Tkachenko oscillations have periods that are larger than their non-dissipative counterparts. In fact, the damping caused by mutual friction is not always strong enough to preclude an oscillatory behavior. Therefore, we conclude that the long term variation in the spin of PSR 1828-11 can in principle be explained in terms of Tkachenko oscillations within superfluid shells for a broad range of values of the mutual friction and the normal fluid shear viscosity. Finally, we note that larger wave vector oscillations corresponding to periodic motions on shorter length scales may lead to phenomena that are responsible for the observed timing noise in pulsars.

Our model necessarily involves certain approximations. 
For instance, we have adopted the two-fluid superfluid hydrodynamics, which should be modified in order to account for the multiple fluids in the neutron star's core [33, 34, 35, 36, 37, 38, 39]. Furthermore, the cylindrical symmetry of our setup and the assumption of uniform density need to be relaxed in more realistic treatments of spherical superfluid shells with density gradients.

\section{ACKNOWLEDGMENTS}

J.N. acknowledges support by the Frankfurt International Graduate School for Science (FIGSS).
[1] J. M. Cordes, in Planets around Pulsars, ed. J. A. Phillips, S. E. Thorsett and S. R. Kulkarni, (ASP, San Francisco, 1993), pg. 43.

[2] F. D'Alessandro, Astrophys. and Space Science 246, 73 (1997).

[3] I. H. Stairs, A. G. Lyne, and S. L. Shemar, Nature 406, 484 (2000).

[4] G. Hobbs, The Discovery and Timing of Radio Pulsars, Ph.D thesis, University of Manchester, 2002.

[5] G. Hobbs, A. Lyne and M. Kramer, Chin. J. Astron. Astrophys. 6, 169 (2006).

[6] T. Akgun, B. Link and I. Wasserman, Mon. Not. Roy. Astron. Soc. 365, 653 (2006) arXiv:astro-ph/0506606.

[7] I. Wasserman, Mon. Not. Roy. Astron. Soc. 341, 1020 (2003) arXiv:astro-ph/0208378.

[8] C. Cutler, G. Ushomirsky and B. Link, Astrophys. J. 588, 975 (2003) arXiv:astro-ph/0210175.

[9] B. Link and R. I. Epstein, Astrophys. J. 556, 392L (2001) arXiv:astro-ph/0101434.

[10] D. I. Jones and N. Andersson, Mon. Not. Roy. Astron. Soc. 324, 811 (2001) arXiv:astro-ph/0011063.

[11] J. Shaham, Astrophys. J. 214, 251 (1977).

[12] A. Sedrakian, I. Wasserman and J. M. Cordes, Astrophys. J. 524, 341 (1999) arXiv:astro-ph/9801188.

[13] B. Link, Phys. Rev. Lett. 91, 101101 (2003).

[14] B. Link, Astron. and Astrophys. 458, 881L (2006); B. Link, Astrophys. Space Sci. 308, 435 (2007).

[15] A. Sedrakian, Phys. Rev. D 71, 083003 (2005).

[16] V. K. Tkachenko, Sov. Phys. JETP 22, 1282 (1966) [Zh. Eksp. Teor. Fiz. 49, 1875].

[17] V. K. Tkachenko, Sov. Phys. JETP 23, 1049 (1966) [Zh. Eksp. Teor. Fiz. 50, 1573].

[18] V. K. Tkachenko, Sov. Phys. JETP 29, 945 (1969) [Zh. Eksp. Teor. Fiz. 56, 1763].

[19] V. K. Tkachenko, 1973, Zh. Eksp. Teor. Fiz. Pis'ma Red., $17,617$.

[20] M. Ruderman, Nature 225, 619 (1970).

[21] P. Donati and P. M. Pizzochero, Phys. Lett. B 640, 74 (2006).

[22] P. Avogadro, F. Barranco, R. A. Broglia and E. Vigezzi, Phys. Rev. C 75, 012805(R) (2007).

[23] A. Sedrakian, Mon. Not. Roy. Astron. Soc. 277, 225 (1995).

[24] B. Link and C. Cutler, Mon. Not. Roy. Astron. Soc. 336, 211 (2002) arXiv:astro-ph/0108281.

[25] L. M. Franco, B. Link and R. I. Epstein, Astrophys. J. 543, 987 (2000) arXiv:astro-ph/9911105.

[26] J. Middleditch, F. E. Marshall, Q. D. Wang, E. V. Gotthelf, and W. Zhang, Astrophys. J. 652, 1531 (2006)

[27] J. Charbonneau and A. Zhitnitsky, Phys. Rev. C 76, 015801 (2007) arXiv:astro-ph/0701308.
[28] M. Alford, G. Good and S. Reddy, Phys. Rev. C 72, 055801 (2005) arXiv:nucl-th/0505025.

[29] D. M. Sedrakian, A. D. Sedrakian and G. F. Zharkov, Mon. Not. Roy. Astron. Soc. 290, 203 (1997) arXiv:astro-ph/9710280.

[30] G. Baym, C. J. Pethick, and D. Pines, Nature 224, 673 (1969).

[31] G. Baym and C. Pethick, Ann. Rev. Nucl. Part. Sci. 25, 27 (1975).

[32] P. Muzikar and C. J. Pethick, Phys. Rev. B 24, 2533 (1981).

[33] G. A. Vardanyan and D. M. Sedrakyan, Sov. Phys. JETP 54, 919 (1981).

[34] G. Mendell, Astrophys. J. 380, 515 (1991); ibid, pg 530.

[35] G. Mendell and L. Lindblom, Ann. Phys. 205, 110 (1991).

[36] G. Mendell, Mon. Not. Roy. Astron. Soc. 296, 903 (1998)

[37] A. D. Sedrakian and D. M. Sedrakian, Astrophys. J. 447, 305 (1995).

[38] A. Sedrakian and J. M. Cordes, Mon. Not. Roy. Astron. Soc. 307, 365 (1999). arXiv:astro-ph/9806042.

[39] N. Andersson and G. Comer, Classical and Quantum Gravity 23, 5505 (2006).

[40] T. Akgun and I. Wasserman, Mon. Not. Roy. Astron. Soc. 383, 1551 (2008).

[41] C. Peralta, A. Melatos, M. Giacobello and A. Ooi, Astrophys. J. 635, 1224 (2005) arXiv:astro-ph/0509416.

[42] C. Peralta, A. Melatos, M. Giacobello and A. Ooi, Astrophys. J. 651, 1079 (2006) arXiv:astro-ph/0607161.

[43] N. Andersson, T. Sidery and G. L. Comer, Mon. Not. Roy. Astron. Soc. 381, 747 (2007) arXiv:astro-ph/0703257.

[44] K. Glampedakis, N. Andersson and D. I. Jones, arXiv:0708.2693 [astro-ph].

[45] A. Melatos and C. Peralta, arXiv:0710.2455 [astro-ph].

[46] I. M. Khalatnikov, An Introduction to the Theory of Superfluidity, (Westview Press, New York, 2000).

[47] G. E. Volovik and V. S. Dotsenko, Sov. Phys. JETP 51, 65 (1980) [Zh. Eksp. Teor. Fiz. 78, 132].

[48] G. Baym and E. Chandler, J. Low Temp. Phys. 50, 57 (1983).

[49] E. Chandler and G. Baym, J. Low Temp. Phys. 62, 119 (1986).

[50] Baym-Chandler hydrodynamics has also been applied to the problem of the spin-up of superfluid He II confined between two parallel plates in A. Reisenegger, J. Low Temp. Phys. 92, 77 (1993).

[51] C. J. Gorter and J. H. Mellink, Physica 15, 285 (1949).

[52] E. Flowers and N. Itoh, Astrophys. J. 230, 847 (1979). 